\title{
LINEAR FRACTIONAL MAPS AND JORDAN ALGEBRAS
}

\author{
KYM S. WATSON
}

\begin{abstract}
Jordan algebras are characterized from amongst the finite dimensional commutative algebras without the use of identities. This is achieved by investigating the properties of the linear fractional transformations generated by the quasi-inversion and all translations.
\end{abstract}

In [3], M. Koecher characterized unital Jordan algebras as homogeneous unital commutative algebras. Some further descriptions may be found in [1]. Here we give another characterization in terms of the linear fractional transformations generated by the quasi-inversion and all translations. This result reinforces the special role played by the linear fractional maps in Jordan algebras.

1. The Lie algebras Rat $V$ and Pol $V$. Let $K$ be an infinite field of characteristic different from 2 and 3 , and $V$ a finite dimensional vector space over $K$. The rational maps $V \rightarrow V$, Rat $V$, and the polynomial maps $V \rightarrow V$, Pol $V$, form Lie algebras over $K$ with the product

$$
[h, k](x)=(d h)_{x} k(x)-(d k)_{x} h(x) \quad(\text { see }[4]) .
$$

Let $D_{i}=D_{i}(V), i=-1,0,1, \ldots$, be the subspace of Pol $V$ of all polynomials homogeneous of degree $i+1$. Then $\left[D_{i}, D_{j}\right] \subset D_{i+j}$ and

$$
\text { Pol } V=\underset{i>-1}{\dot{+}} D_{i}(V)
$$

is a graded decomposition of Pol $V$. We will identify $D_{-1}$ with $V$.

2. The quasi-inversion. Let $A$ be a finite dimensional commutative algebra over $K$. By adjoining a unit element $e$ to $A$ we can form a new commutative algebra with inversion. The quasi-inversion is the unique birational map $q$ in Rat $A$ satisfying

$$
(e+x)(e+q(x))=e .
$$

Then $q^{2}=\mathrm{id}, q(0)=0$, and $\left(d^{n} q\right)_{0}(x, \ldots, x)=(-1)^{n} n ! x^{n}$ where $x^{n+1}=$ $x x^{n}$ for $n=1,2, \ldots$ Define the rational map $Q: A \rightarrow$ End $A$ by $Q(x)=$ $-(d q)_{x}^{-1}$.

Received by the editors May 11, 1977.

AMS (MOS) subject classifications (1970). Primary 17C99, 17A99, 17B60; Secondary 53A40. Key words and phrases. Jordan algebra, linear fractional map, quasi-inversion. 


\section{Linear fractional maps.}

Definition. Let $G(A)$ be the group of birational transformations of $A$ generated by the quasi-inversion $q$ and all translations $T_{a}(x)=a+x, a \in$ A.

If $A$ has a unit element $e_{0}$, with inversion $j$, then $q=T_{-e_{0}} j T_{e_{0}}$ and $G(A)$ is generated by $j$ and all translations.

Definition. (a) Let $L_{1}(A)$ be the Lie subalgebra of Rat $A$ generated by all the $h \in$ Rat $A$ such that id $+\delta h \in G\left(A \otimes_{K} K[\delta]\right)$ where $K[\delta]$ is the algebra of dual numbers over $K\left(\delta^{2}=0\right)$.

(b) Let $L_{2}(A)$ be the Lie subalgebra of Rat $A$ generated by $D_{-1}(A)$ and all $Q(x) a, a \in A$.

\section{Characterizations of Jordan algebras.}

THEOREM. Let $A$ be a commutative finite dimensional algebra over $K$. Then the following statements are equivalent:

(1) $A$ is a Jordan algebra.

(2) $L_{1}(A)$ is a subspace of $D_{-1} \dot{+} D_{0} \dot{+} D_{1}$.

(3) $L_{2}(A)$ is a subspace of $D_{-1}+D_{0} \dot{+} D_{1}$.

(4) $Q(x)$ is a polynomial map $A \rightarrow$ End $A$ of degree 2 or less.

Proof. (1) $\Rightarrow(2)$. For every finite dimensional Jordan algebra $A$ over $K$, consider the Lie subalgebra $L(A)$ of $\operatorname{Pol} A$ generated by $D_{-1}(A)$, id and $v(x)=x^{2}$. Then $L(A)$ is well known to be the linear span of all constant maps $x \rightarrow a, a \in A$, all quadratic polynomial maps of the form $x \rightarrow 2 x(x a)$ $-x^{2} a, a \in A$, all linear maps of the form $x \rightarrow b(x a)+x(b a)-(b x) a, a$, $b \in A$, and the identity map (see [2] for a proof; the assumption made there that $K$ be the reals is not used). Hence $L(A)$ is a binary Lie algebra in the sense of [4]. We will use the results of [4].

Let Birat $A$ be the group of birational transformations of $A$. For every $f \in$ Birat $A$ and $h \in$ Rat $A$, define $h^{f} \in$ Rat $A$ by

$$
h^{f}(x)=(d f)_{x}^{-1} h(f(x))
$$

Now for every $f \in$ Birat $A$ define the automorphism $\nabla_{f}$ of Rat $A$ by

$$
\nabla_{f}(h)=h^{f^{-1}}
$$

and hence $\nabla$ is a monomorphism from Birat $A$ into the group of automorphisms of Rat $A$ (see [4, Kapitel I, §1,6]).

We claim that $\nabla_{f}$ maps $L(A)$ into itself for all $f \in G(A)$. It suffices to prove this for $f=T_{a}, a \in A$, and for $f=q$, the quasi-inversion of $A$.

If $f=T_{a}$, then $\nabla_{f}$ maps $L(A)$ into itself by [4, Kapitel I, $\left.\S 3,2\right]$.

Let $A^{\prime}$ be the unital Jordan algebra obtained by adjoining a unit element $e$ to $A$. For $x, y \in A^{\prime}$, let $P(x) y=2 x(x y)-x^{2} y$. Define $w \in$ Birat $A$ by 
$w(x)=-x$. For $x \in A$,

$$
\begin{aligned}
(q \circ w)(x) & =(e-x)^{-1}-e \\
& =P(e-x)^{-1}(e-x-P(e-x) e) \quad\left(\text { as } P(y)^{-1} y=y^{-1}\right) \\
& =P(e-x)^{-1}\left(x-x^{2}\right) .
\end{aligned}
$$

A simple calculation shows that $P(e-x)=B_{v}(x)$ on $A$ where

$$
B_{v}(x) a=\left(a-[v, a]+\frac{1}{2}[v,[v, a]]\right)(x)
$$

for $a, x \in A$. Hence $(q \circ w)(x)=B_{v}(x)^{-1}(x-v(x))$ and $\nabla_{q \circ w}$ maps $L(A)$ into itself by [4, Kapitel II, Lemma 1.2]. Now $\nabla_{w}(h)=w \circ h \circ w^{-1}$ for $h \in$ Rat $A$. Hence $\nabla_{w}$ maps $L(A)$ into itself. Therefore $\nabla_{q}=\nabla_{q \circ w} \circ \nabla_{w}$ also maps $L(A)$ into itself.

Now let $h \in$ Rat $A$ be such that $f=$ id $+\delta h \in G\left(A \otimes_{K} K[\delta]\right)$. Then by the above $\nabla_{f}$ maps $L\left(A \otimes_{K} K[\delta]\right)$ into itself. Note that the given description of the Lie algebras $L(B)$ for Jordan algebras $B$ shows that $L\left(A \otimes_{K} K[\delta]\right)$ is naturally isomorphic to $L(A) \otimes_{K} K[\delta]$. Since

$$
\left(\nabla_{f}(p)\right)(x)=\left(\mathrm{id}+\delta(d h)_{x}\right) p(x-\delta h(x))=p(x)+\delta[h, p](x)
$$

for $p \in L(A) \subset L\left(A \otimes_{K} K[\delta]\right)$ and for $x \in A$, it follows that $[h, L(A)] \subset$ $L(A)$. Hence $p \rightarrow[h, p]$ is a derivation of $L(A)$ for all $h \in L_{1}(A)$. By [4, Kapitel II, Satz 3.1] there exists, for each $h \in L_{1}(A), h^{\prime} \in D_{-1}(A)+D_{0}(A)$ $\dot{+} D_{1}(A)$ such that $\left[h-h^{\prime}, L(A)\right]=0$. We will now show that if $h \in$ Rat $A$ is such that $[h, L(A)]=0$, then $h=0$. From this and from the above remarks, it will then follow that $L_{1}(A)$ is a subspace of $D_{-1}(A) \dot{+} D_{0}(A)$ $\Varangle D_{1}(A)$.

Let $h \in$ Rat $A$ be such that $[h, L(A)]=0$. Then

$$
(d h)_{x}(a)=[h, a](x)=0
$$

for all $a \in A$. So from

$$
0=[h, \mathrm{id}](x)=(d h)_{x}(x)-h(x)
$$

we can deduce that $h=0$.

$(2) \Rightarrow(3)$. Since $T_{a \delta}(x)=x+a \delta$ and $q T_{-\delta a} q(x)=x+\delta Q(x) a$, then $L_{2}(A)$ is a subalgebra of $L_{1}(A)$.

(3) $\Rightarrow(4)$. This is evident.

(4) $\Rightarrow(1)$. Let $g(x)=(d q)_{x}(a)$. Then, since $g \circ q(x)=-Q(x) a$,

$$
\begin{aligned}
0= & \left(d^{3}(g \circ q)\right)_{0}(x, x, x) \\
= & \left(d^{3} g\right)_{0}\left((d q)_{0}(x),(d q)_{0}(x),(d q)_{0}(x)\right) \\
& +3\left(d^{2} g\right)_{0}\left(\left(d^{2} q\right)_{0}(x, x),(d q)_{0}(x)\right)+(d g)_{0}\left(\left(d^{3} q\right)_{0}(x, x, x)\right) \\
= & -\left(d^{4} q\right)_{0}(x, x, x, a)-6\left(d^{3} q\right)_{0}\left(x^{2}, x, a\right)-6\left(d^{2} q\right)_{0}\left(x^{3}, a\right) \\
= & -6\left(a x^{3}+x\left(a x^{2}\right)+2 x(x(x a))\right) \\
& +6\left(2 a x^{3}+2 x^{2}(a x)+2 x\left(a x^{2}\right)\right)-6\left(2 a x^{3}\right) .
\end{aligned}
$$


Hence

$$
0=a x^{3}-x\left(a x^{2}\right)+2 x(x(x a))-2 x^{2}(a x) .
$$

Set $a=x$ and deduce that $x^{4}=x^{2} x^{2}$. Differentiate the last equation to obtain $a x^{3}+x\left(a x^{2}\right)+2 x(x(x a))=4(x a) x^{2}$. Finally, add this equation to $(*): x\left(x^{2} a\right)=x^{2}(x a)$ as required.

THEOREM. Let $A$ be a finite dimensional unital commutative algebra over $K$ with inversion $j$. Then the following statements are equivalent.

(1) $A$ is a Jordan algebra.

(2) $(d j)_{x}^{-1}$ is a polynomial map $A \rightarrow$ End $A$.

Moreover if char $K=0$, then (1) and (2) are each equivalent to

(3) $L_{1}(A)$ is finite dimensional.

(4) $L_{2}(A)$ is finite dimensional.

Proof. (1) $\Rightarrow(2)$. This is well known [1].

(2) $\Rightarrow(1)$. Since $q=T_{-e} j T_{e}$, where $e$ is the unit element of $A$, then $Q(x)=-(d j)_{x+e}^{-1}$ is a polynomial map $A \rightarrow$ End $A$ of degree 2 . The previous theorem now implies that $A$ is a Jordan algebra. Assume now that char $K=$ 0 .

(1) $\Rightarrow(3)$. This was done above.

$(3) \Rightarrow(4)$. This is clear since $L_{2}(A)$ is a subspace of $L_{1}(A)$.

$(4) \Rightarrow(2)$. The rational maps $p_{n}(x)=\left[\left(d^{n} Q\right)_{x}\left(a_{1}, \ldots, a_{n}\right)\right](y), \quad n=$ $1,2, \ldots$, belong to $L_{2}(A)$ as they are formed by successively commutating by $a_{i}$, starting with $Q(x) y$. Moreover, $p_{n}(x-e)$ is homogeneous of degree $2-n$. Hence, for some $m, p_{n}(x)=0$ for all $a_{1}, \ldots, a_{n} \in A$ and for all $n \geqslant m$. Since char $K=0, Q(x)$ is a polynomial map $A \rightarrow$ End $A$. Therefore $(d j)_{x}^{-1}$ is a polynomial map $A \rightarrow \operatorname{End} A$.

5. A counterexample. Let $A$ be a finite dimensional commutative algebra over $K$ in which any product of $n$ elements is zero. Then $q(x)=$ $\sum_{m=1}^{n-1}(-1)^{m} x^{m},(d q)_{x}^{-1}$ is a polynomial map $A \rightarrow \operatorname{End} A$, and $L_{2}(A)$ is finite dimensional, but $A$ need not be a Jordan algebra.

\section{REFERENCES}

1. H. Braun and M. Koecher, Jordan-Algebren, Springer-Verlag, Berlin, 1966.

2. I. L. Kantor, Transitive differential groups and invariant connections on homogeneous spaces, Trudy Sem. Vektor. Tenzor. Anal. 13 (1966), 310-398. (Russian)

3. M. Koecher, On homogeneous algebras, Bull. Amer. Math. Soc. 72 (1966), 347-357.

4. __ Gruppen und Lie-Algebren von rationalen Funktionen, Math. Z. 109 (1969), 349-392.

School of Mathematical Sciences, Flinders University, Bedford Park, 5042, South Australia

Department of Pure Mathematics, The Australian National University, Canberra, A.C.T. 2600, Australia (Current address) 